\title{
Depressive Cognitions May Affect Cingulate Neurochemistry in ADHD Patients
}

\author{
Evgeniy Perlov ${ }^{1}$, Alexandra Philipsen ${ }^{1}$, Simon Maier ${ }^{1}$, Martin Buechert ${ }^{2}$, Bernd Hesslinger ${ }^{1}$, \\ Dieter Ebert ${ }^{1}$, Ludger Tebartz van Elst ${ }^{1}$ \\ ${ }^{1}$ University Hospital Freiburg, Department of Psychiatry and Psychotherapy, Hauptstr, Freiburg, Germany \\ ${ }^{2}$ University Hospital Freiburg, Department of Diagnostic Radiology, Hugstetterstr, Freiburg, Germany \\ E-mail: evgeniy.perlov@uniklinik-freiburg.de \\ Received December 29, 2010; revised January 28, 2011; accepted February 14, 2011
}

\begin{abstract}
Objectives: The anterior cingulate is thought to be essentially involved in impulsivity and affect regulation and in the pathogenesis of depression as well as of attention deficit/hyperactivity disorder. At the same time alterations in glutamatergic neurotransmission in the frontal forebrain have been found in imaging studies in Attention Deficit Hyperactivity Disorder (ADHD) and in depressive patients. Therefore we hypothesized that glutamate/glutamine (Glx) signals in the anterior cingulate cortex (ACC) of depressed ADHD patients might differ from that in non-depressed patients. Methods: Fourteen male adult patients with ADHD were included into the study. Chemical Shift Imaging of ACC was performed. Subgroups were defined based on scores on the Beck Depression Inventory (BDI) and ratios of metabolites were compared between groups. Additionally correlation analyses of BDI scores with metabolite ratios were calculated. Results: Significantly lower Glx signals and N-acetyl-aspartate (NAA) signals were found in the left anterior cingulate cortex of depressed ADHD patients. The Glx/Cr and NAAX/Cr ratios in the left ACC correlated significantly with BDI-scores. Conclusions: To our knowledge this is the first report about a relationship between depressive symptoms and metabolite disturbances in ACC of adult patients with ADHD. Our preliminary data produce first evidence for a putative link between neurochemical alterations in the ACC and depressive symptoms. They should be controlled for in further studies.
\end{abstract}

Keywords: ADHD, MR-Spectroscopy, Depression, Anterior Cingulum, Glutamate

\section{Introduction}

Most psychiatric disorders are known to be overrepresented in ADHD patients throughout the life from childhood into adulthood. Psychiatric comorbidities include alcohol and other substance abuse as well as mood and anxiety disorders and borderline and antisocial personality disorders [1-4] . Co morbidities with differential types of depression - major depressive disorder (MDD), dysthymia and brief recurrent depression - are particularly common [5]. This might be due to underachievement in school, further education and professional life as well as to frequent interpersonal problems in the family and relationships. Depression in ADHD patients is often atypical. Anhedonia and physiological concomitants of depression are often absent whereas restlessness and inattention seem to be dominant $[1,6]$.
Dopaminergic disturbance is thought to be a crucial pathogenetic mechanism in ADHD [7,8]. Additionally, glutamatergic alterations in the prefrontal cortex have been thought to be involved in the pathogenesis of this disorder in children [9,10] as well as in adults [11]. At the same time glutamatergic disturbances in prefrontal cortex and in particular in the anterior cingulated cortex (ACC) have been reported in MDD [12,13] and in some disorders in association with depressive symptoms [14,15].

Proton-Magnet-Resonanz-Spectroscopy $\left({ }^{1} \mathrm{H}-\mathrm{MRS}\right)$ is a non-invasive method which allows to detecting signals from neurometabolites in vivo. Amongst others N-acetylaspartate (NAA), N-acetylaspartate-glutamine (NAAG) - taken together - NAAx, creatine and phosphocreatine (Cre), choline compounds (Cho), glutamate and glutamine (Glx) and myo-inositol (Ins) can be measured with 
${ }^{1} \mathrm{H}$-MRS [16].

Based on the observations summarized above we wanted to analyse in this study if glutamate signals in the prefrontal cortex are altered in relation to symptoms of depression in adult patients with ADHD.

\section{Methods}

Approval from the local ethics committee was obtained before onset of the study.

The data presented here are part of larger and ongoing project at the University Hospital of Freiburg in which we attempt to define the cross-sectional and longitudinal neuroanatomy and neurochemistry in adult ADHD patients [Freiburg ADHD Imaging Study in Adults (FAISA)] and had been partly reported elsewhere [11,17].

Fourteen male ADHD patients (age $31.0 \pm 11.0$ ) from our out-patient department were included in this study. The previous and current medication experiences and comorbidity of other psychiatric (including major depression) and neurological diseases which may affect brain metabolism served as exclusion criteria for this study. That means that none of the subjects included did suffer from clincally relevant depressive disorder according to DSM-IV or ICD-10 criteria. Psychometric assessment included the Wender Utah Rating Scale (WURS [18]; German version [19]) and the ADHD check list, corresponding to the DSM-IV criteria. All the patients suffered from combined subtype of ADHD. Subliminal symptoms of depression at time of scanning were assessed using the Beck Depression Inventory (BDI)
[20].

Spectroscopic data and anatomical 3D datasets were obtained on a 1.5 Tesla MR-scanner (Magnetom Sonata, Siemens Erlangen, Germany). The method of Chemical Shift Imaging (CSI) was employed with the following parameters: $\mathrm{TR}=1670 \mathrm{~ms}, \mathrm{TE}=3.9 \mathrm{~ms}, \mathrm{TI}=1100 \mathrm{~ms}$, flip angle $=15$ degrees, matrix $256 \times 256$ pixel, FOV = $256 \times 256 \mathrm{~cm}^{2}$. The previously published LC-algorithm [21] in combination with the CSILcmodel-Tool [22] was used for postprocessing of the data, the Cramer-Row lower bound values smaller then $20 \%$ were used as a quality criterion. The regions of interest (ROI) covering the right and left ventral ACC were placed manually by a neuroanatomically trained rater (EP) in a blinded way. Only selected parts of the ROI without contamination with CSF were included. Ratios of metabolites (NAA, Cho, Glx and Ins) to Cre were calculated following established procedures in LC-Model (Figure 1).

The software program SPSS 13.0 was used for statistical analysis. Subgroups with respect to subliminal depressive symptoms ( $\mathrm{n}=7$ for each) were defined by median split of the overall group (13) based on BDI-scores. Multiple analysis of covariance (MANCOVA) with metabolites to Cre ratios (Glx, NAA, Cho, Ins) as dependant variables and age, scores of ADHD-CL and WURS as covariates were chosen independently for the right and left ACC. Additionally a Pearson correlation coefficient for BDI scores and metabolite signals was calculated. A p-value of 0.05 was chosen here as the criterion of significance.

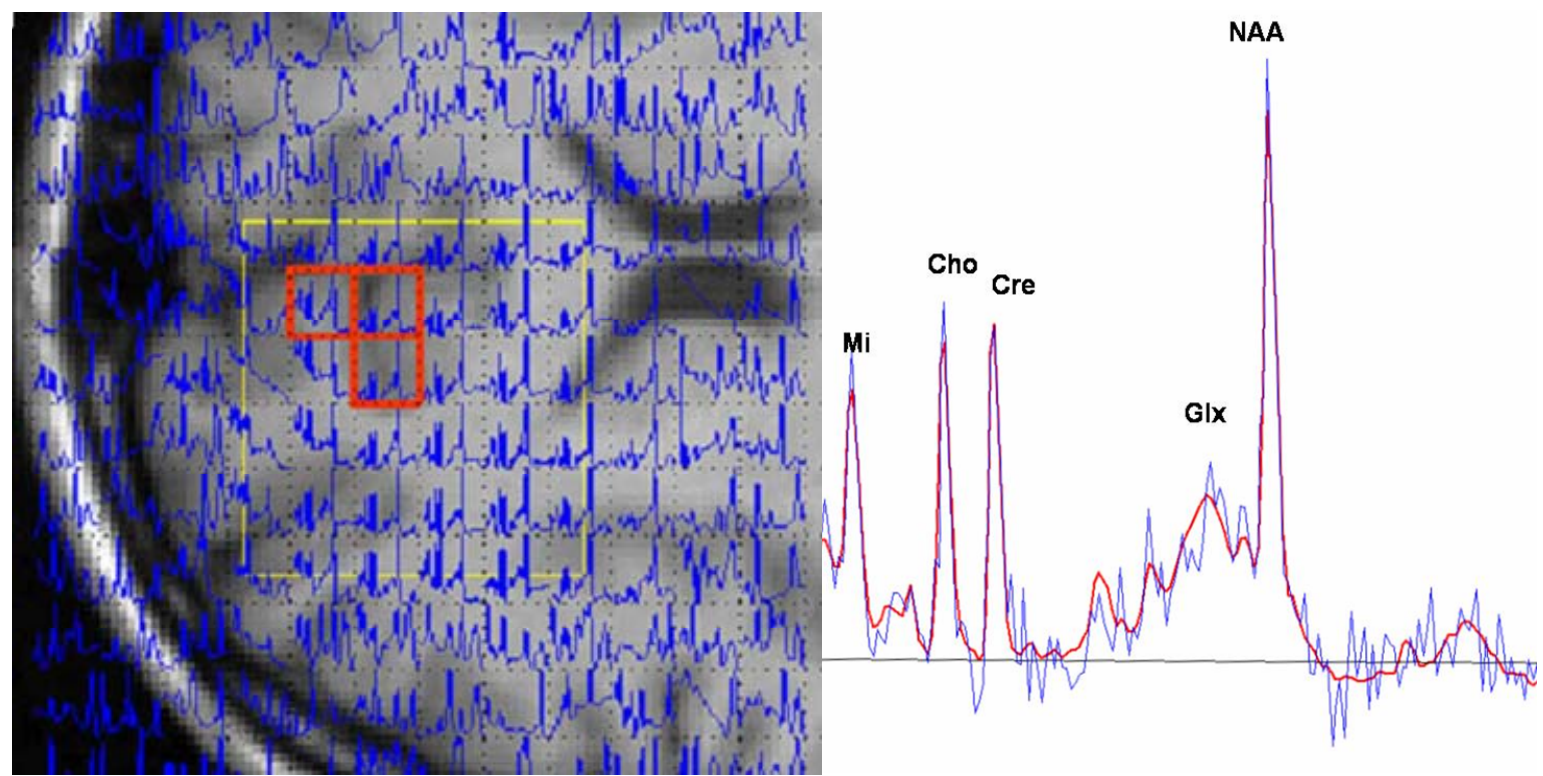

[MRS = Magnet Resonance Spectroscopy, Cre $=$ Creatine, NAA $=$ N-acetylaspartate, Cho $=$ Choline, $\mathrm{mI}=$ Myoinositol, Glx $=$ Glutamate + Glutamine $]$

Figure 1. CSI-voxels localization on a superposition of the MRS slice onto the anatomical data set (in red - selected voxels) and MR spectrum of the anterior cingulum. 


\section{Results}

The groups did not differ with respect to age, education and values of WURS and ADHD-CL scores (Table 1).

We found a significant influence of the factor group (ADHD patients with higher versus lower BDI scores) in multivariate Wilks-Lambda-test for the left ( $\mathrm{F}=7.515$; $\mathrm{df}=5.00 ; \mathrm{p}=0.024)$ but not for the right $(\mathrm{F}=0.37$; $\mathrm{df}=$ 5.00; $p=0.822$ ) ACC. MANCOVA revealed that the more depressive patients with ADHD displayed signifi- cant reductions in NAA and Glx signals in the left ACC. There were no significant differences with respect to other MRS signals (Table 2)

The correlation analysis revealed a significant negative correlation between the BDI-scores and Glx/Cr ratios $(\mathrm{r}=0.610 ; \mathrm{p}=0.02)$ and NAA/Cr ratios $(\mathrm{r}=0.654 ; \mathrm{p}$ $=0.01)$ in the left ACC. There were no significant correlations of BDI-scores and any other metabolite concentration in the right or left ACC. The scatter plots of the correlation analyses are presented in Figures $\mathbf{2}$ and $\mathbf{3}$.

Table 1. Patient assessment.

\begin{tabular}{|c|c|c|c|c|}
\hline & $\begin{array}{l}\text { Complete } \\
\mathrm{n}=14\end{array}$ & $\begin{array}{c}\text { Depressive } \\
n=7\end{array}$ & $\begin{array}{l}\text { Non-depressive } \\
\qquad n=7\end{array}$ & Statistics \\
\hline Age & $31.0 \pm 11.0$ & $27.2 \pm 5.8$ & $32.4 \pm 10.1$ & $t=1.196 ; d f=9.55 ; p=0.255$ \\
\hline Years of school education & $12.4 \pm 1.3$ & $12.1 \pm 1.5$ & $12.6 \pm 1.1$ & $\mathrm{t}=0.612 ; \mathrm{df}=11.29 ; \mathrm{p}=0.552$ \\
\hline WURS & $62.4 \pm 10.8$ & $61.4 \pm 12.7$ & $63.4 \pm 9.3$ & $\mathrm{t}=0.335 ; \mathrm{df}=11.01 ; \mathrm{p}=0.744$ \\
\hline ADHD-CL & $25.5 \pm 4.4$ & $25.1 \pm 5.7$ & $26.4 \pm 2.8$ & $t=0.535 ; d f=8.64 ; p=0.606$ \\
\hline BDI & $13.3 \pm 5.7$ & $17.7 \pm 2.4$ & $8.9 \pm 4.4$ & $\mathrm{t}=-4.682 ; \mathrm{df}=9.38 ; \mathrm{p}=0.001$ \\
\hline
\end{tabular}

Spectroscopic findings

Table 2. Spectroscopic findings.

\begin{tabular}{|c|c|c|c|c|c|}
\hline ROI & Metabolite/Cr & Side & $\begin{array}{l}\text { More depressive } \\
\qquad n=7\end{array}$ & $\begin{array}{l}\text { Less depressive } \\
\qquad n=7\end{array}$ & Statistics \\
\hline \multirow{8}{*}{ ACC } & \multirow{2}{*}{ NAAx } & left & $1.33 \pm 0.11$ & $1.53 \pm 0.22$ & $\mathrm{~F}=5.004 ; \mathrm{df}=1 ; \mathrm{p}=0.045$ \\
\hline & & right & $1.41 \pm 0.21$ & $1.38 \pm 0.25$ & not significant \\
\hline & \multirow{2}{*}{ Cho } & left & $0.31 \pm 0.04$ & $0.34 \pm 0.02$ & not significant \\
\hline & & right & $0.32 \pm 0.02$ & $0.32 \pm 0.03$ & not significant \\
\hline & \multirow{2}{*}{ Ins } & left & $0.85 \pm 0.17$ & $0.79 \pm 0.11$ & not significant \\
\hline & & right & $0.89 \pm 0.13$ & $0.84 \pm 0.05$ & not significant \\
\hline & \multirow{2}{*}{ Glx } & left & $1.62 \pm 0.23$ & $2.22 \pm 0.64$ & $F=5.283 ; \mathrm{df}=1 ; \mathrm{p}=0.040$ \\
\hline & & right & $1.61 \pm 0.29$ & $1.79 \pm 0.51$ & not significant \\
\hline
\end{tabular}

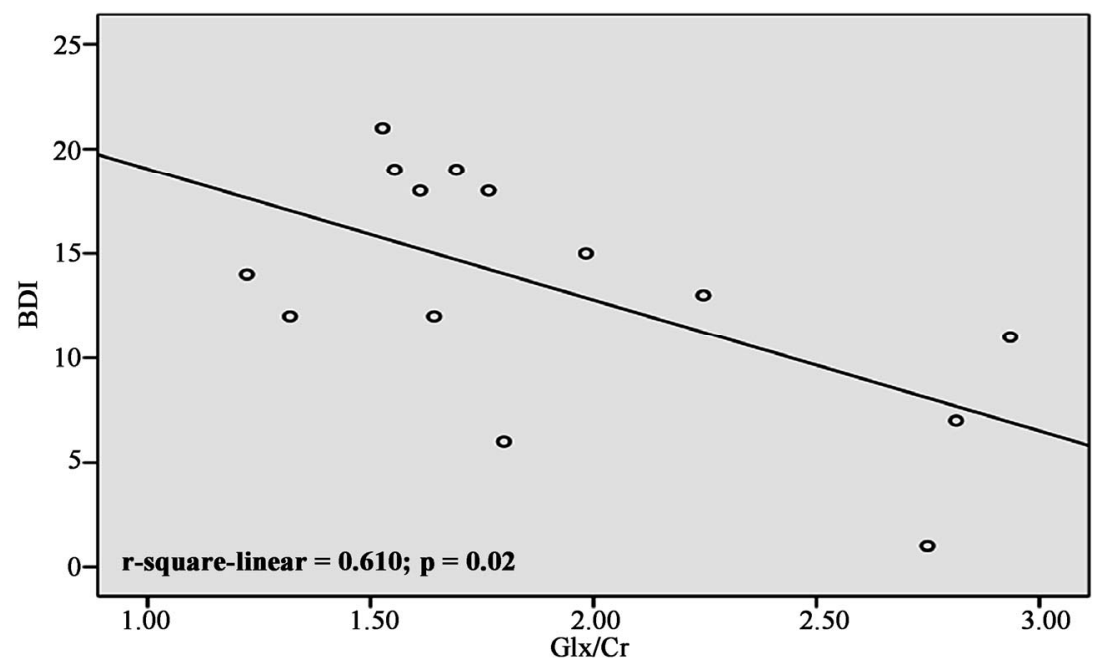

Figure 2. Correlation between BDI-value and Glx/Cr ratio in left ACC. 


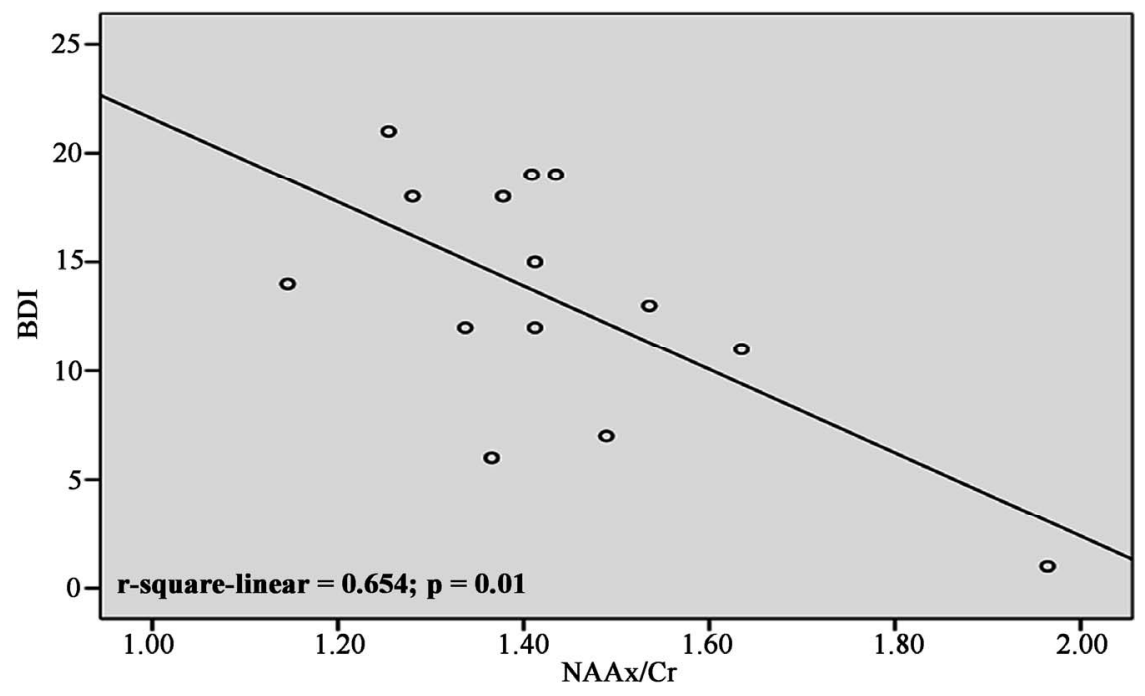

Figure 3. Correlation between BDI-value and NAAx/Cr ratio in left ACC.

We found no significant correlation between WURSor ADHD-CL-scores and any neurometabolite.

\section{Discussion}

In this pilot study we specifically tested the hypothesis of an influence of subliminal depressive symptoms as measured with BDI on the glutamatergic neurochemistry in adult male patients with ADHD. We did find preliminary evidence for alterations in glutamatergic metabolism and, additionally, in NAA signals, both in the left ACC in the group of patients with depressive symptoms. Supporting the noting of a pathogenetic link between depressive symptoms and left ACC NAA and Glx metabolism, we found a significant and rather strong correlation between Glx/Cr and NAAX/Cr ratios in the left ACC and BDI-scores.

To our knowledge this is the first report of such a link between ACC Glx signals and depressive symptoms in adult ADHD. The prefrontal glutamatergic neurons modulate the release of other neurotransmitters including dopamine and serotonin in the midbrain [23]. The dopaminergic system is well known to be important in the pathogenesis of ADHD. The dopaminergic neurotransmission of course is of critical importance for depression. Furthermore, in our recent study we reported lower Glx-signals in the ACC of adult patients with ADHD compared to healthy controls [11]. On the other hand there is an increasing body of evidence for disturbed glutamatergic neurotransmission in MDD and depressive comorbidity in medical disorders like for example diabetes mellitus [12-15]. NAA, a marker of functional or structural integrity of the neurons, has also been reported to be altered in ADHD but not in MDD [5,24].

There are many limitations to this pilot study. First of all the number of included subjects is very small. Second, the results have been obtained only in male subjects and therefore cannot be generalized to females. Most importantly, there is no control group of healthy subjects or patients with depressive symptoms without ADHD and for that reason we cannot conclude if or not this association might be restricted to ADHD patients or not. The precise meaning of altered Glx or NAA signals with respect to specific pathophysiological mechanisms are also not yet resolved.

Therefore, these findings have to been taken with caution and should be regarded as preliminary evidence or hypothesis-generating evidence which does need replication.

Nevertheless, we feel that when looking at the raw data with respect to group differences and correlation analyses the signal seems to be rather strong and valid. Therefore, further research should aim at establishing if or not there is an association between left ACC glutamate metabolism and if this is restricted to subliminal symptoms or ADHD patients only. If this relationship could be established for depressive symptoms in general irrespective of severity and neuropsychiatric comorbidity such a signal could be of relevance as possible neurobiological marker of depressive symptoms in general. Further studies in ADHD patients should analyze possible relationships between depressive symptoms and neurochemical findings. Vice versa, further MRS studies in ADHD should be controlled for possible influence of subclinical depressive symptoms on glutamatergic signal in order to avoid the misinterpretation of the findings.

\section{Acknowledgments}

None. 


\section{References}

[1] J. Biederman, S. V. Faraone, T. Spencer, T. Wilens, E. Mick and K. A. Lapey, "Gender Differences in a Sample of Adults with Attention Deficit Hyperactivity Disorder," Psychiatry Research, Vol. 53, No. 1, 1994, pp. 13-29. doi:10.1016/0165-1781(94)90092-2

[2] J. E. Alpert, A. Maddocks, A. A. Nierenberg, R. O'Sullivan, J. A. Pava, J. J. Worthington, J. Biederman, J. F. Rosenbaum and M. Fava, "Attention Deficit Hyperactivity Disorder in Childhood among Adults with Major Depression,” Psychiatry Research, Vol. 62, No. 3, 1996, pp. 213-219. doi:10.1016/0165-1781(96)02912-5

[3] R. A. Barkley, M. Fischer, L. Smallish and K. Fletcher, "The Persistence of Attention-Deficit/Hyperactivity Disorder into Young Adulthood as a Function of Reporting Source and Definition of Disorder," Journal of Abnormal Psychology, Vol. 111, No. 2, 2002, pp. 279-289. doi:10.1037/0021-843X.111.2.279

[4] T. E. Wilens, "Impact of ADHD and Its Treatment on Substance Abuse in Adults," Journal of Clinical Psychiatry, Vol. 65, No. 3, 2004, pp. 38-45.

[5] B. Hesslinger, T. Thiel, L. T. van Elst, J. Hennig and D. Ebert, "Attention-Deficit Disorder in Adults with and without Hyperactivity - Where is the Difference? A Study Using Short Echo 1H-magnetic-resonance Spectroscopy,” Neuroscience Letters, Vol. 304, No. 1, 2001, pp. 117-119. doi:10.1016/S0304-3940(01)01730-X

[6] B. Hesslinger, v. E. Tebartz, F. Mochan and D. Ebert, “A Psychopathological Study into the Relationship between Attention Deficit Hyperactivity Disorder in Adult Patients and Recurrent Brief Depression," Acta Psychiatrica Scandinavica, Vol. 107, No. 5, 2003, pp. 385-389. doi:10.1034/j.1600-0447.2003.00112.x

[7] D. D. Dougherty, A. A. Bonab, T. J. Spencer, S. L. Rauch, B. K. Madras and A. J. Fischman, "Dopamine Transporter Density in Patients with Attention Deficit Hyperactivity Disorder,” Lancet, Vol. 354, No. 9196, 1999, pp. 2132-2133. doi:10.1016/S0140-6736(99)04030-1

[8] S. V. Faraone, J. Biederman, T. Spencer, T. Wilens, L. J. Seidman, E. Mick and A. E. Doyle, "Attention-Deficit/ Hyperactivity Disorder in Adults: An Overview," Biological Psychiatry, Vol. 48, No. 1, 2000, pp. 9-20. doi:10.1016/S0006-3223(00)00889-1

[9] F. P. MacMaster, N. Carrey, S. Sparkes and V. Kusumakar, "Proton Spectroscopy in Medication-free Pediatric Attention-Deficit/Hyperactivity Disorder,” Biological Psychiatry, Vol. 53, No. 2, 2003, pp. 184-187. doi:10.1016/S0006-3223(02)01401-4

[10] C. M. Moore, J. Biederman, J. Wozniak, E. Mick, M. Aleardi, M. Wardrop, M. Dougherty, T. Harpold, P. Hammerness, E. Randall and P. F. Renshaw, "Differences in Brain Chemistry in Children and Adolescents with Attention Deficit Hyperactivity Disorder with and without Comorbid Bipolar Disorder: A Proton Magnetic Resonance Spectroscopy Study," American Journal of Psychiatry, Vol. 163, No. 2, 2006, pp. 316-318.

doi:10.1176/appi.ajp.163.2.316
[11] E. Perlov, A. Philipsen, B. Hesslinger, M. Buechert, J. Ahrendts, B. Feige, E. Bubl, J. Hennig, D. Ebert and v. E. Tebartz, "Reduced Cingulate Glutamate/Glutamine-toCreatine Ratios in Adult Patients with Attention Deficit/hyperactivity Disorder - A Magnet Resonance Spectroscopy Study," Journal of Psychiatric Research, Vol. 41, No. 11, 2007, pp. 934-941. doi:10.1016/j.jpsychires.2006.12.007

[12] M. Walter, A. Henning, S. Grimm, R. F. Schulte, J. Beck, U. Dydak, B. Schnepf, H. Boeker, P. Boesiger and G. Northoff, "The Relationship between Aberrant Neuronal Activation in the Pregenual Anterior Cingulate, Altered Glutamatergic Metabolism, and Anhedonia in Major Depression,” Archives of General Psychiatry, Vol. 66, No. 5, 2009, pp. 478-486. doi:10.1001/archgenpsychiatry.2009.39

[13] D. P. Auer, "Reduced Glutamate in the Anterior Cingulate Cortex in Depression: An in vivo Proton Magnetic Resonance Spectroscopy Study,” Biological Psychiatry, Vol. 47, No. 4, 2000, pp. 305-313. doi:10.1016/S0006-3223(99)00159-6

[14] I. K. Lyoo, S. J. Yoon, G. Musen, D. C. Simonson, K. Weinger, N. Bolo, C. M. Ryan, J. E. Kim, P. F. Renshaw and A. M. Jacobson, "Altered Prefrontal GlutamateGlutamine-Gamma-Aminobutyric Acid Levels and Relation to Low Cognitive Performance and Depressive Symptoms in Type 1 Diabetes Mellitus," Archives of General Psychiatry, Vol. 66, No. 8, 2009, pp. 878-887. doi:10.1001/archgenpsychiatry.2009.86

[15] P. Ohrmann, A. Kersting, T. Suslow, J. Lalee-Mentzel, U. S. Donges, M. Fiebich, V. Arolt, W. Heindel and B. Pfleiderer, "Proton Magnetic Resonance Spectroscopy in Anorexia Nervosa: Correlations with Cognition,” NeuroReport, Vol. 15, No. 3, 2004, pp. 549-553. doi:10.1097/00001756-200403010-00033

[16] B. Ross and S. Bluml, "Magnetic Resonance Spectroscopy of the Human Brain,” Anatomical Record, Vol. 265, No. 2, 2001, pp. 54-84. doi:10.1002/ar.1058

[17] E. Perlov, v. E. Tebarzt, M. Buechert, S. Maier, S. Matthies, D. Ebert, B. Hesslinger and A. Philipsen, "H(1)MR-spectroscopy of Cerebellum in Adult Attention Deficit/Hyperactivity Disorder," Journal of Psychiatric Research, Vol. 44, No. 1414, 2010, pp. 938-943.

[18] M. F. Ward, P. H. Wender and F. W. Reimherr, "The Wender Utah Rating Scale: An Aid in the Retrospective Diagnosis of Childhood Attention Deficit Hyperactivity Disorder," American Journal of Psychiatry, Vol. 150, No. 6, 1993, pp. 885-890.

[19] K. H. Krause, J. Krause and G. E. Trott, "Hyperkinetic Syndrome (Attention Deficit/Hyperactivity Disorder) in Adulthood," Nervenarzt, Vol. 69, No. 7, 1998, pp. 543-556. doi:10.1007/s001150050311

[20] A. T. Beck, R. A. Steer and M. G. Carbin, "Psychometric Properties of the Beck Depression Inventory: Twenty-five Years of Evaluation,” Clinical Psychology Review, Vol. 8, No. 1, 1988, pp. 77-100. doi:10.1016/0272-7358(88)90050-5

[21] S. W. Provencher, "Estimation of Metabolite Concentra- 
tions from Localized in vivo Proton NMR Spectra,” Magnetic Resonance in Medicine, Vol. 30, No. 6, 1993, pp. 672-679. doi:10.1002/mrm.1910300604

[22] C. W. Ko, B. Kreher and M. Buchert, "GUI for Automatic Post Processing and Display of 2D-SI Data Sets with LC Model,” ISMRM, 11th Annual Meeting, 2003.

[23] T. A. Slotkin, E. C. McCook, J. C. Ritchie, B. J. Carroll and F. J. Seidler, "Serotonin Transporter Expression in Rat Brain Regions and Blood Platelets: Aging and Glu- cocorticoid Effects,” Biological Psychiatry, Vol. 41, No. 2, 1997, pp. 172-183.

doi:10.1016/S0006-3223(96)00215-6

[24] A. Yildiz-Yesiloglu and D. P. Ankerst, "Review of $1 \mathrm{H}$ Magnetic Resonance Spectroscopy Findings in Major Depressive Disorder: A Meta-analysis,” Psychiatry Research, Vol. 147, No. 1, 2006, pp. 1-25. doi:10.1016/j.pscychresns.2005.12.004 